\title{
Towards the construction of a model to describe the inter-ELM evolution of the pedestal on MAST
}

\author{
D. Dickinson ${ }^{1,2}$, S. Saarelma ${ }^{2}$, R. Scannell ${ }^{2}$, \\ A. Kirk ${ }^{2}$, C.M. Roach ${ }^{2}$ and H.R. Wilson ${ }^{1}$
}

November 6, 2018

1 York Plasma Institute, Dept of Physics, University of York, Heslington, York, YO10 $5 \mathrm{DD}, \mathrm{UK}$

${ }^{2}$ Euratom/CCFE Fusion Association, Culham Science Centre, Abingdon, OX14 3DB, UK

\begin{abstract}
Pedestal profiles that span the ELM cycle have been obtained and used to test the idea that the pedestal pressure gradient in MAST is limited by the onset of Kinetic Ballooning Modes (KBMs). During the inter-ELM period of a regularly type I ELM-ing discharge on MAST, the pressure pedestal height and width increase together while the pressure gradient increases by only $15 \%$ during the ELM cycle. Stability analyses show that the pedestal region over which infinite-n ballooning modes are unstable also broadens during the ELM cycle. To test the relationship between the width of the region that is unstable to $n=\infty$ ideal magnetohydrodynamic ballooning modes and KBMs the gyrokinetic code, GS2, has been used for microstability analysis of the edge plasma region in MAST. The gyrokinetic simulations find that KBM modes with twisting parity are the dominant microinstabilities in the steep pedestal region, with a transition to tearing parity modes in the shallower pressure gradient core region immediately inside the pedestal top. The region over which KBMs are unstable increases during the ELM cycle, and a good correlation is found between the region where KBMs dominate and the region that is unstable to infinite-n ideal ballooning modes.
\end{abstract}




\section{Introduction}

The reference scenario for ITER [1] is the high confinement mode (or H-mode) [2]. The improved confinement is the result of a narrow transport barrier that forms at the plasma edge. Due to the strong coupling between pedestal and core confinement, which has been observed in many devices [3], the fusion performance of ITER will strongly depend on the achievable pedestal pressure. Therefore, an accurate prediction of the pedestal height is essential for the prediction and optimization of ITER performance. A class of instabilities called Edge Localized Modes or ELMs [4], [5] limits the maximum pressure pedestal height, $P_{\text {ped }}$, that can be achieved for a given pedestal width. Hence, understanding the width of the barrier is very important in determining the maximum pressure that can be achieved. An MHD stability analysis has been used to show that in order for ITER to attain a temperature pedestal of $4 \mathrm{keV}$, which is thought to be required for ITER to attain its fusion power output objective, the pedestal width would have to be $2.5 \%$ of the minor radius [6], [7]. Several empirical scalings for the pedestal width $\left(\Delta_{\text {ped }}\right)$ have been proposed based on dimensionless parameters such as the normalized poloidal ion Larmor radius $\left(\rho_{\text {pol }}^{*}\right)$ or pressure $\left(\beta_{\text {pol }}\right)$, but a strong co-linearity between pedestal values of $\beta_{\text {pol }}$ and $\rho_{\text {pol }}^{*}$ limits the power of these datasets to discriminate between very different

scalings: e.g. early data from DIII-D could be fit equally well with either $\Delta_{\text {ped }}=\left(\rho_{\text {pol }}^{*}\right)^{0.66}$ or $\Delta_{\text {ped }}=\left(\beta_{\text {pol }}\right)^{0.4}$ [8]. Experiments on JT-60U using hydrogen and deuterium plasmas found a scaling of the form $\Delta_{\text {ped }} \propto\left(\rho_{\mathrm{pol}}^{*}\right)^{0.2}\left(\beta_{\mathrm{pol}}\right)^{0.5}[9$. Recent similarity experiments on DIII-D, ASDEX Upgrade and JET also obtain a pedestal width scaling, in normalised poloidal flux, of the form $\Delta_{\Psi_{N}} \propto \beta_{\mathrm{pol}}^{0.5}$ [10]. Previous studies on MAST have shown no evidence for an increase of the width with $\rho_{\text {pol }}^{*}$ but a clear increase of width with $\beta_{\text {pol }}$ [11]. Fits of the distributions of MAST pedestal width measurements to the form $\Delta_{\text {ped }}=A \beta_{\text {pol }}^{B}$ yielded $B \approx 0.5$. A similar scaling of the pedestal width has also been observed on DIII-D, C-MOD and AUG [12]. The scaling $\Delta_{\text {ped }} \propto \beta_{\text {pol }}^{0.5}$ has been used to provide basic input to the EPED model for pedestal evolution [13]. The EPED model proposes that: drift wave turbulence is suppressed in the pedestal region by sheared flow; kinetic ballooning mode (KBM) turbulence constrains the pedestal to a critical normalized pressure gradient; and an ELM is triggered by the onset of the intermediate $\mathrm{n}(\sim 10-20)$ peeling-ballooning mode instability. This model provides a prediction for the pressure pedestal width and height just before the ELM crash. EPED was successfully compared to pedestal conditions in DIII-D and a range of other tokamaks [12,,[13]. The model has since been extended to exploit the fact that KBMs are predicted to have an onset condition similar to that of ideal infinite-n ballooning modes [14], where $n$ is the toroidal mode number. In the more recent version of EPED, the infinite-n ideal MHD ballooning mode code, BALOO [15], has been used to compute the threshold pressure gradient set by the condition that either $1 \%$ of the pressure profile in normalized poloidal flux [14 or $50 \%$ of the steep pedestal gradient region [16] is unstable to infinite-n ideal ballooning modes. This technique has found good agreement between the predicted and observed pedestal widths and heights obtained prior to type I ELMs on DIII-D [14] and JET [17].

In this paper, the ideas incorporated in the EPED model have been tested using data from the spherical tokamak MAST. In order to construct a pedestal model for MAST, the evolution of the pedestal profiles has been studied as a function of time during the 
inter-ELM period, and this is described in Section 2. In Section 3 the HELENA code [18] has been used to reconstruct equilibria during the ELM cycle and to determine infinite-n ideal ballooning stability, and the ELITE code [19, 20] has been used to calculate the finite-n stability boundary. In Section 4 the local gyrokinetic code, GS2 [21], has been used to perform linear microstability analysis in the pedestal region and to test whether the region unstable to infinite-n ballooning modes is related to the region where KBMs are unstable in MAST (i.e. to test whether ideal infinite-n MHD ballooning analysis can be used as a proxy for KBM stability in MAST).

\section{Inter-ELM pressure gradient evolution on MAST}

The data examined in this paper were obtained from a set of three MAST discharges \#24459, \#24452 and \#24763. All discharges were in a double null configuration with a plasma current of $850 \mathrm{kA}$, a toroidal magnetic field on axis of $0.585 \mathrm{~T}$ and heated by 3.4 MW of neutral beam injection. The timeslices examined were obtained during the inter-ELM period of a regularly type I ELM-ing discharge.

The data used in this paper were taken from a Thomson scattering system with 130 spatial points [22, 23]. This system combines eight Nd:YAG lasers and measures $\mathrm{T}_{e}$ and $\mathrm{n}_{e}$ profiles approximately every $4.2 \mathrm{~ms}$. This sampling rate is not normally sufficient to diagnose an individual ELM period (6-12ms), but arranging the profiles obtained over a number of plasmas as a function of time with respect to the ELM cycle allowed the general cycle of periodic ELMs to be diagnosed. Each spatial point in the Thomson scattering system has a scattering length of $10 \mathrm{~mm}$. At the Low Field Side (LFS) edge of the plasma this results in $10 \mathrm{~mm}$ radial resolution, $\Delta_{R}$, as the laser path is in an approximately radial direction. Close to the High Field Side (HFS) edge of the plasma, the laser is at an angle to the radial direction and this results in much higher $(3-5 \mathrm{~mm})$ radial resolution.

Measured edge profiles were fitted to an analytic parameterisation, $P(r ; \vec{a})$, convolved with the instrument function to account for the spatial resolution of the diagnostic. $P(r ; \vec{a})$ is based on a modified hyperbolic tangent function, mtanh, and is defined [24]:

$$
P(r ; \vec{a})=\frac{a_{\text {ped }}-a_{\text {sol }}}{2}\left[\operatorname{mtanh}\left(\frac{a_{\text {etb }}-r}{2 a_{\Delta}}, a_{\text {slope }}\right)+1\right]+a_{\text {sol }},
$$

where

$$
\operatorname{mtanh}(r, a)=\left(\frac{(1+a r) e^{r}-e^{-r}}{e^{r}+e^{-r}}\right)
$$

using as fit parameters: pedestal height, $a_{\text {ped }}$; scrape off layer height, $a_{\text {sol }}$; transport barrier position, $a_{\mathrm{etb}}$; width, $a_{\Delta}$; and core slope, $a_{\text {slope }}$. The pedestal width for profile quantity $a, \Delta_{a}$, is determined from these parameters as $\Delta_{a}=4 a_{\Delta}$. Electron temperature and density pedestal width measurements on the LFS were typically close to the limits of spatial resolution, giving $\Delta_{\mathrm{Te}, \mathrm{ne}} / \Delta_{R} \sim 1-2$ : but on the HFS there were sufficient data points in the pedestal region to reduce the uncertainties, giving $\Delta_{T e, n e} / \Delta_{R} \sim 3-4$. All data examined in this paper were therefore obtained from measurements at the HFS of MAST. 
Three profiles obtained during a single ELM cycle are shown in figure 1, The $\mathrm{T}_{e}(\mathrm{r})$ and $\mathrm{n}_{e}(\mathrm{r})$ profiles were first fitted in real space using the parameterisation of equation (1) convolved with the instrument response function. These fitted profiles were then mapped to normalised poloidal flux space $\left(\Psi_{N}\right)$, where a further fit was used to obtain pressure profile fit parameters in $\Psi_{N}$. The electron pressure gradient, $d P_{e} / d \Psi_{N}$, was computed from this fit.

During the ELM cycle examined in figure 1, the change in the temperature profile is small. The density profile shows a large change, with $\mathrm{n}_{e, p e d}$ and $\Delta_{n e}$ increasing throughout the ELM cycle. The resulting pressure pedestal change, dominated by the growing density pedestal, shows increasing $P_{e, p e d}$ and expanding $\Delta_{P e}$. The pressure gradient profile evolution during the ELM cycle shows an expanding region of high pressure gradient (with little change in the peak pressure gradient), indicating that a transport barrier is expanding inwards towards the core.

Examining the peak pressure gradient obtained in this manner over several inter-ELM periods, and plotting the result as a function of time during the ELM cycle (exploiting the fact that the ELMs are periodic), results in the data shown in figure 2. From this graph we see that immediately after the ELM, the drop in pressure gradient recovers rapidly during the first $10 \%$ of the ELM cycle. During the remainder of the cycle there is only a weak upwards trend in the peak pressure gradient.

A more complete picture of the pedestal evolution in the inter-ELM period was obtained by combining the 50 profiles from the three MAST discharges. For each timeslice, the mtanh fit parameters for electron density, electron temperature and electron pressure profiles in $\Psi_{N}$ space, have been obtained as previously described. Least squares linear fits were found to represent well the time evolution of each mtanh parameter over the ELM cycle. Such linear fits were used to evaluate the mtanh profile parameters at five normalised times during the ELM cycle, $t=(0.1,0.3,0.5,0.7,0.9)$. Table 1 gives the interpolated values of the mtanh fit parameters for the electron pressure profile at these times, and figure 3 shows the reconstructed electron profiles. The peak electron pressure gradient from each of these profiles is compared with the peak electron pressure gradients determined directly from mtanh fits to individual profiles in figure 2,

Figures 3(a) and (b) show little change in the electron temperature pedestal height, whilst $\Delta_{T e}, \Delta_{n e}$ and the density pedestal height increase during the ELM cycle. It can be seen from figure 3 (c) that the electron pressure pedestal height and width increase during the ELM cycle. Equilibrium reconstruction shows little change in the flux expansion at the HFS during the ELM cycle, and therefore the $\Delta_{p e}$ expansion in real space tracks that in $\Psi_{N}$ space. The calculated electron pressure gradient is shown in figure 3(d). The increase in $\mathrm{P}_{e, p e d}$ over the ELM cycle is mainly associated with the expansion in $\Delta_{p e}$, and only partly with a modest increase in the pressure gradient, which increases by $<20 \%$ over the ELM cycle. The high pressure gradient pedestal region expands during the ELM cycle: the innermost surface where $d p / d \Psi_{N}>10 \mathrm{kPa}$ moves inwards from $\Psi_{N}=0.975$ at $t=0.1$ to $\Psi_{N}=0.95$ at $t=0.9$, while the location of the peak pressure gradient also moves inwards from $\Psi_{N}=0.988$ at $t=0.1$, to $\Psi_{N}=0.978$ at $t=0.9$ (where $t$ denotes normalised time during the ELM cycle). Similar observations were made during regular type I ELM-ing periods in other plasma scenarios on MAST, DIII-D [14] and AUG [25]. 


\section{MHD Stability Limits During the ELM Cycle}

Measured electron temperature and density profiles were used to reconstruct MHD equilibria to describe the edge plasma with high precision. Five such equilibria were produced to span the ELM cycle. Pressure profiles were obtained from the $n_{e}$ and $T_{e}$ profiles of figure 3 and model profiles for ion temperature and density, as ion measurements were not available with sufficient temporal and spatial resolution. A uniform effective charge state, $Z_{\text {eff }}=2$, 1 was assumed, for a plasma with Carbon as the main impurity species so that $n_{i}=n_{e}\left(7-Z_{e f f}\right) / 6$. Two ion temperature models were adopted: $T_{i}=T_{e}$ across the whole plasma including the pedestal region; and setting $T_{i}=T_{e}$ in the core, but extrapolating $T_{i}$ from the pedestal top to the separatrix using the electron temperature gradient from the core side of the pedestal top, to avoid a steep edge gradient in $T_{i}$. The plasma boundary shape, total plasma current and vacuum magnetic field were taken from MAST equilibrium reconstructions using EFIT [26]. The inductively driven current profile was assumed to be fully relaxed, and the bootstrap current, which dominates at the edge, was calculated using the formula by Sauter et al. [27], [28]. These assumptions have allowed the reconstruction of high precision equilibria using the HELENA code [18]. These equilibria exploit the available constraints from the TS measurements self-consistently, and were used for the MHD and gyrokinetic stability analyses presented here and in Section 4 , respectively.

In order to determine the limiting pressure gradients for ideal MHD instability, scans in the equilibrium pedestal pressure gradient have been performed, either by scaling the temperature pedestal height and gradient (at constant pedestal width) keeping the density profile fixed, or by scaling the density pedestal height and gradient (at constant pedestal width) keeping the temperature profile fixed. Both approaches gave similar results, but here results from the latter approach are presented, as this is closer to the experimental situation. The equilibrium scan parameter is the maximum value, $\alpha_{\max }$, of the normalised pressure gradient, $\alpha$, given by [29]:

$$
\alpha=\frac{-2 \partial V / \partial \psi}{(2 \pi)^{2}}\left(\frac{V}{2 \pi^{2} R_{0}}\right)^{1 / 2} \mu_{0} \frac{\partial p}{\partial \psi},
$$

where $p$ is the pressure, $V$ is the volume enclosed by the flux surface, $R_{0}$ is the geometric centre of the plasma and $\psi$ is the poloidal flux. In scans the pedestal pressure gradient was varied by up to a factor 2.5 (while the equilibrium stored energy varied by $<30 \%$ ), and the bootstrap current was always calculated self-consistently for each equilibrium using the appropriately modified density and temperature profiles.

Ideal MHD stability analysis was performed using two codes, depending on the toroidal mode number $n$. The HELENA code used for equilibrium reconstruction, was also used to determine infinite-n ideal ballooning stability on each flux surface [18]. The ELITE code [19, 20] was used to analyse stability of intermediate $n$ modes. In this study the finite $n$ modes were restricted to a maximum value of $n=25$, which roughly corresponds to the maximum $n$ observed in experimental measurements of ELM filaments in MAST [30]. The lowest $n$ considered was $n=5$.

\footnotetext{
${ }^{1} Z_{\text {eff }}=2$ is consistent with typical experimental values in the MAST pedestal. The core $Z_{\text {eff }}$ is typically in the range 1-1.5.
} 
All five experimental equilibria are unstable to infinite-n ideal ballooning modes. The radial region that is unstable to $n=\infty$ modes is plotted in figure 4 as a function of normalised time in the ELM cycle, and will be compared to the region unstable to KBMs in Section 4. Infinite-n ballooning stability was not found to be sensitive to the choice of ion temperature model in the pedestal region. All other MHD and gyrokinetic stability analyses were also insensitive to the ion temperature model, so the remaining stability results presented in this paper assumed $T_{i}=T_{e}$ throughout the plasma.

Figure 5 shows the growth rate of the most unstable intermediate $n$ ideal MHD mode, $n=25$, as a function of $\alpha_{\max }$, for scans around the first and last equilibria in the ELM cycle. The threshold for the finite-n instability, $\alpha_{\mathrm{c}}$, corresponds to the $\alpha_{\max }$ value above which the mode becomes unstable with a rapidly rising growth rate. Figure 5 illustrates that while the experimental pedestal pressure gradient, $\alpha_{\exp }$, is far below $\alpha_{\mathrm{c}}$ at the start of the ELM cycle, it is slightly above $\alpha_{\mathrm{c}}$ by the end of the cycle.

Figure 3 shows that the maximum pressure gradient increases only slightly during the ELM cycle: the maximum of the edge bootstrap current density $(\propto \nabla p)$ also changes very little. The main change during the ELM cycle is the broadening of the pedestal. Figure 6 plots $\alpha_{\exp }$ and $\alpha_{\mathrm{c}}$ as functions of normalised time during the ELM cycle. While the experimental pressure gradient changes very little, the stability limit is lowered significantly as the pedestal broadens. This can be understood in terms of stabilising finite $n$ corrections in ballooning theory [31], which are larger for the equilibria with narrower pedestals from earlier in the ELM cycle. It has frequently been found useful to present the proximity of equilibria to edge localised MHD stability boundaries using plots of MHD stability versus edge pressure gradient and current density $\left(\alpha_{\max }, j_{\phi}\right)$ [32. Such diagrams have considerable value, but their description of stability dynamics over the full ELM cycle is limited by the difficulty of capturing the full equilibrium evolution in a simple 2-parameter model.

Figure 7 shows the experimental pressure pedestal height as a function of time during the ELM cycle. Superimposed on this plot are the stability limits derived from the infinite-n ideal ballooning and finite- $n$ stability analyses. The finite- $n$ stability limit is well above the experimental value at the start of the ELM cycle, but later it converges on the experimental value just before an ELM, which is consistent with the theory that type I ELMs are triggered by an ideal finite- $n$ instability. A stability criterion, based on $100 \%$ of the pedestal region 2 being unstable to infinite-n ideal MHD ballooning modes, tracks the experimental pedestal pressure height to within 20\% throughout the MAST ELM cycle. Pedestal pressure gradient limits derived from criteria that the region unstable to $n=\infty$ ballooning modes be wider than (i) $1 \%$ of the total poloidal flux, or (ii) $50 \%$ of the pedestal, however, lie well below the experimental pedestal pressure throughout the cycle.

In addition to analysing the stability of equilibria during the ELM cycle, an analysis similar to that used in the EPED model [13] was repeated. Edge stability was determined for a range of equilibria, using a scan around the reference equilibrium just prior to the ELM crash. In this scan the density and temperature pedestal widths and the density pedestal height were scaled together around their measured values, keeping the density

\footnotetext{
${ }^{2}$ The width of the pedestal region is defined: $\Delta_{\text {ped }}=\left(\Delta_{n_{e}}+\Delta_{T_{e}}\right) / 2$.
} 
gradient constant. Then, for each pedestal width $\Delta_{\text {ped }}$, the temperature pedestal height was varied to determine stability boundaries. Figure 8 plots various stability limits as functions of the scan parameter $\Delta_{\text {ped }}$ : the finite- $n$ limit; and limits based on $1 \%$ of the poloidal flux, and $50 \%$ and $100 \%$ of the pedestal, respectively, being unstable to infinite-n ideal ballooning modes. The pedestal top pressure limit due to finite $n$ modes increases with pedestal width, but more slowly than the scanned pedestal top pressure (which is $\propto \Delta_{\text {ped }}$ if the pressure gradient is constant). The limit requiring $100 \%$ of the pedestal to be unstable to $n=\infty$ ideal ballooning modes, crosses the finite $n$ limit close to the experimental pedestal width just before an ELM. While this could in principle provide an EPED-like model for the pedestal height and width in MAST 3 , the figure of $100 \%$ has no theoretical justification. It will be necessary to understand this parameter to make predictions for future devices.

\section{Microstability analysis during the ELM cycle}

Kinetic descriptions can improve on MHD models of edge instabilities, and the gyrokinetic model is exploited here. The H-mode pedestal is associated with a strong pressure gradient that can drive electromagnetic instabilities, like the KBM, where the magnetic perturbations are crucial to the instability mechanism. Experimental measurements suggest that the KBM may play a very important role in the H-mode edge, by setting a hard limit on the maximum pressure gradient achievable in the pedestal region [14]. Early work has shown that collisionless KBMs and ideal MHD infinite-n ballooning modes are described by related equations, and that these modes have similar character [33]. This suggests that it can be reasonable to use the stability of the infinite-n ballooning mode as a proxy for KBM stability. Nevertheless, finite Larmor radius (FLR) effects, only included in the kinetic treatment, are often stabilising [34, and the region unstable to infinite-n ideal MHD ballooning modes can be significantly broader than the region unstable to KBMs. The importance of FLR effects can be measured by the parameter $\Lambda$ :

$$
\Lambda=\frac{\left(k_{y} \rho_{i}\right)^{2} R}{2 L_{P}}
$$

where $L_{P}$ is the pressure gradient scale length, $k_{y}$ is the perturbation wavenumber in the flux surface and perpendicular to magnetic field lines, and $\rho_{i}$ is the ion Larmor radius. When $\Lambda \ll 1$ (corresponding to $L_{P} \gg\left(k_{y} \rho_{i}\right)^{2} R$ ), FLR effects are negligible and the KBM stability limit is close to that of the ideal infinite-n ballooning mode: when $\Lambda \sim O(1)$, however, FLR effects become significant and should not be neglected [34]. This suggests that the ideal infinite-n calculation can only describe KBM stability consistently in regions of shallow pressure gradient, and raises questions as to the validity of using infinite-n ideal ballooning stability as a proxy for KBM stability in the pedestal region.

In order to investigate this, a local electromagnetic gyrokinetic analysis has been used to obtain the microstability properties of the MAST edge plasma during the ELM cycle.

\footnotetext{
${ }^{3}$ A pedestal evolution model cannot be constructed for MAST using a criterion based on either $1 \%$ of poloidal flux [14] or $50 \%$ of the pedestal [16] being unstable to infinite-n ideal ballooning modes, as the experimental pressure profile appears to be well above this limit.
} 
The gyrokinetic model is based on a first order expansion in the small parameter $\delta=\rho_{i} / L$ (where $L$ is a typical equilibrium gradient scalelength), which includes FLR corrections that are missing from ideal MHD. Clearly gyrokinetics should be less accurate in the pedestal than in the core, as the pedestal value of $\delta$ is larger owing to shorter equilibrium gradient scale lengths. Using the MAST data at the time corresponding to the fully developed pedestal (i.e. just prior to the ELM crash), we obtain $\delta \sim 0.3$ (ie $\delta<1$ ) at $\Psi_{N}=0.98$, which corresponds to the surface at the mid-radius in the fully developed pedestal. At this location it is interesting to note that $\Lambda \sim 1$ for $k_{y} \rho_{i} \sim 0.2$, so FLR corrections should be important for $k_{y} \rho_{i} \geq 0.2$. While conditions in the pedestal limit the accuracy of the gyrokinetic model, gyrokinetics is more appropriate than MHD as it includes FLR corrections.

The local gyrokinetic code, GS2, has been used to perform linear microstability analysis around the pedestal during the MAST ELM cycle, based on the data described in Section 2 and the equilibria prepared for MHD stability analysis in Section 3. GS2 is an initial value code, and finds, for specified values of $k_{y} \rho_{i}$, the fastest growing microinstability and provides its growth rate, $\gamma$. GS2 was used to obtain the dominant growth rate spectra, which were captured in the range $0.07<k_{y} \rho_{i}<5.5$, at 12 radial locations between $\Psi_{N}=0.94$ and $\Psi_{N}=0.995$, for 5 times during the ELM cycle, $t=(0.1,0.3,0.5,0.7,0.9)$. The characteristics of the dominant mode reveal the radial region where KBMs are expected to dominate, and this can be compared directly with the region found to be unstable to infinite-n ideal ballooning modes using HELENA.

Figure 9(a) shows the parity factor, $C_{\mathrm{par}}$, of the dominant mode in the $k_{y}$ range $0.07<k_{y} \rho_{i}<5.5$, and figure 9(b) shows the growth rate of the fastest growing mode at $k_{y} \rho_{i}=0.218$, which corresponds to the wavenumber of the dominant KBM instability over the ELM cycle; both quantities are illustrated as functions of radius and time through the ELM cycle. The parity factor is defined:

$$
C_{\mathrm{par}}=1-\frac{\left|\int A_{\|} d \theta\right|}{\int\left|A_{\|}\right| d \theta}
$$

where $A_{\|}$is the parallel magnetic vector potential. The dominant modes are found to be KBMs with twisting parity $\left(C_{\mathrm{par}}=1\right)$ in the steep pressure gradient region closer to the edge, with a transition to electron temperature gradient driven tearing parity modes $\left(C_{\mathrm{par}}=0\right)$ in the shallower gradient core region inside the top of the pedestal. The switch in mode parity is illustrated in figure 10, which presents the dominant eigenfunctions of the electrostatic potential, $\Phi$, and $A_{\|}$, on the $\Psi_{N}=0.98$ and $\Psi_{N}=0.95$ surfaces at $t=0.5$. Microtearing modes have previously been reported to be unstable at mid-radius in MAST plasmas [35, [36], close to the edge in AUG plasmas [37], and in a conceptual fusion power plant based on the spherical tokamak [38].

The infinite-n ideal ballooning unstable region (from figure 44) is also indicated on figure 9(a), and this corresponds closely to the region where KBMs are the dominant modes. This suggests that it is reasonable here to use infinite-n ballooning calculations as a proxy for the dominance of KBMs, even though $\Lambda$ is finite. The KBM unstable region broadens and expands inwards with the strong pressure gradient, as the pedestal width increases through the ELM cycle. Figure 9(b) shows that the KBM growth rate peaks in the steep gradient region close to the top of the pedestal. This peak moves inwards as 
the pedestal expands, and the KBM growth rate reduces at this location after the transit of the peak.

\section{Conclusions}

Pedestal profiles of electron temperature and electron density, obtained as a function of time during the MAST ELM cycle, have been used to test whether the pressure pedestal gradient in MAST is limited by the onset of Kinetic Ballooning Modes (KBMs). In this set of discharges, the electron density and electron pressure pedestal heights and widths increase during the inter-ELM period, while their gradients remain approximately constant. Finite-n stability analyses using the ELITE code show that the finite-n stability limited normalised pressure gradient, $\alpha_{\mathrm{c}}$, lies above the experimental value, $\alpha_{\exp }$, initially just after an ELM, and then falls towards it just before the next ELM: i.e. rather than the experimental pressure gradient increasing towards a fixed stability boundary it is the pressure gradient stability limit that moves towards the experimental pressure gradient due to the increasing pedestal width. The region over which infinite-n modes are unstable also broadens during the ELM cycle. Ideal MHD stability boundary calculations track the evolution of the pedestal height and width well, provided the limiting criterion used is that $100 \%$ of the pedestal width be unstable to infinite-n modes.

The gyrokinetic code, GS2, was used to test whether the MAST pedestal region unstable to KBMs corresponds closely to the region that is unstable to infinite-n ideal ballooning modes. KBM modes with twisting parity were found to be the dominant microinstabilities in the steep pedestal region, with a transition to tearing parity modes in the shallower pressure gradient core region immediately inside the pedestal top. The region over which KBMs dominate increases during the ELM cycle, and closely corresponds to the region unstable to infinite-n ideal ballooning modes. The impact of sheared flow on stability has not been considered here, and an assessment of its possible impact is underway.

\section{Acknowledgement}

This work, part-funded by the European Communities under the contract of Association between EURATOM and CCFE, was carried out within the framework of the European Fusion Development Agreement. The views and opinions expressed herein do not necessarily reflect those of the European Commission. This work was also part-funded by the RCUK Energy Programme under grant EP/I501045. Gyrokinetic simulations were

performed on the supercomputer, HECToR, which was made available through EPSRC grant EP/H002081/1. 


\section{References}

[1] ITER Physics Expert Group 1999 Nucl. Fusion 392391.

[2] Wagner F et al 1982 Phys. Rev. Lett. 491408.

[3] Ryter F et al 2001 Plasma Phys. Control. Fusion 43 A32338

[4] Connor J W 1998 Plasma Phys. Control. Fusion 40531

[5] Suttrop W 2000 Plasma Phys. Control. Fusion 42 A1

[6] Osborne T H et al 2002 19th IAEA Fusion Energy Conf. (Lyon, France, 14/19 October 2002)

[7] Snyder P B et al 2004 Nucl. Fusion 44320

[8] Osborne T H et al 1998 Plasma Phys. Control. Fusion 40845

[9] Urano H et al 2008 Nucl. Fusion 48045008

[10] Beurskens M N A et al, 2011, to be published in Phys Plasmas.

[11] Kirk A et al 2009 Plasma Phys. Control. Fusion 51065016

[12] Snyder P B et al. 2009 Nucl. Fusion 49085035

[13] Snyder P B et al 2009 Phys. Plasmas 16056118

[14] Groebner R J et al 2010 Nucl. Fusion 50064002

[15] Miller R L et al 1997 Phys. Plasmas 41062

[16] Snyder P B, Groebner R J, Hughes J W et al. 2011 Nucl. Fusion 51103016.

[17] Beurskens M et al 2009 Plasma Phys. Control. Fusion 51124051

[18] Huysmans G T A, Goedbloed J P, Kerner W O K 1991 Computational Physics (Proc. Int. Conf. Amsterdam, 1991), World Scientific Publishing, Singapore 371

[19] Wilson H R, Snyder P B, Huysmans G T A and Miller R L 2002 Phys. Plasmas 9 1277

[20] Snyder P B, Wilson H R, et al 2002 Phys. Plasmas 92037

[21] Kotschenreuther M, Rewoldt G and Tang W M 1995 Comp. Phys. Comm. 88128

[22] Scannell R et al 2008 Rev. Sci. Instrum. 79 10E730

[23] Scannell R et al 2010 Rev. Sci. Instrum. 81 10D520

[24] Scannell R et al 2011 Rev. Sci. Instrum. 82053501 
[25] Burckhart A et al 2010 Plasma Phys. Control. Fusion 52105010

[26] Lao L L and St John H and Stambaugh R D and Kellman A G and Pfeiffer W et al, 1985 Nucl. Fusion 251611

[27] Sauter O, Angioni C, Lin-Liu Y R 1999 Phys. Plasmas, 62834

[28] Sauter O, Angioni C, Lin-Liu Y R 2002 Phys. Plasmas 95140

[29] Miller R L et al 1998 Phys. Plasmas 5973

[30] Kirk A et al 2005 Plasma Phys. Control. Fusion 47315

[31] Connor J W, Hastie R J and Taylor J , 1979 Proc. R. Soc. Lond. A365 1

[32] Connor J W, Hastie R J, Wilson H R and Miller R L, 1998 Phys. Plasmas 52687

[33] Tang W M, Connor J W and Hastie R. J., 1980 Nucl. Fusion 201439

[34] Tang W M, Connor J W and White R B, 1981 Nucl. Fusion 21891

[35] Applegate D J and Roach C M and Cowley S C and Dorland W and Hastie R J and Joiner N, 2004 Phys. Plasmas 115085

[36] Applegate D J and Roach C M and Connor J W and Cowley S C and Dorland W and Hastie R J and Joiner N, 2007 Plasma Phys. Control. Fusion 491113

[37] Told D, Jenko F, Xanthopoulos P, Horton L D, Wolfrum E and ASDEX Upgrade Team, 2008 Phys. Plasmas 15102306

[38] Wilson H R et al, 2004 Nucl. Fusion 44, 917 


$\begin{array}{lccccc}t & P_{\text {e,etb }}\left(\psi_{N}\right) & 100 P_{\mathrm{e}, \Delta}\left(\psi_{N}\right) & P_{\mathrm{e}, \mathrm{ped}}(\mathrm{Pa}) & P_{\mathrm{e}, \text { slope }}(\mathrm{Pa}) & P_{\mathrm{e}, \text { sol }}(\mathrm{Pa}) \\ 0.1 & 0.988 \pm 0.0009 & 0.527 \pm 0.027 & 557.5 \pm 40.9 & 0.167 \pm 0.010 & 0.56 \pm 0.31 \\ 0.3 & 0.986 \pm 0.0008 & 0.606 \pm 0.026 & 682.5 \pm 39.1 & 0.166 \pm 0.009 & 0.47 \pm 0.29 \\ 0.5 & 0.983 \pm 0.0009 & 0.686 \pm 0.029 & 807.6 \pm 43.4 & 0.164 \pm 0.011 & 0.38 \pm 0.30 \\ 0.7 & 0.981 \pm 0.0011 & 0.766 \pm 0.035 & 932.7 \pm 52.3 & 0.162 \pm 0.013 & 0.29 \pm 0.34 \\ 0.9 & 0.979 \pm 0.0014 & 0.846 \pm 0.042 & 1058 . \pm 63.9 & 0.160 \pm 0.016 & 0.20 \pm 0.40\end{array}$

Table 1: The five mtanh fit parameters for electron pressure at $t=(0.1,0.3,0.5,0.7,0.9)$, with uncertainties from the least squares linear fits to the evolution of these parameters during the inter-ELM period. 

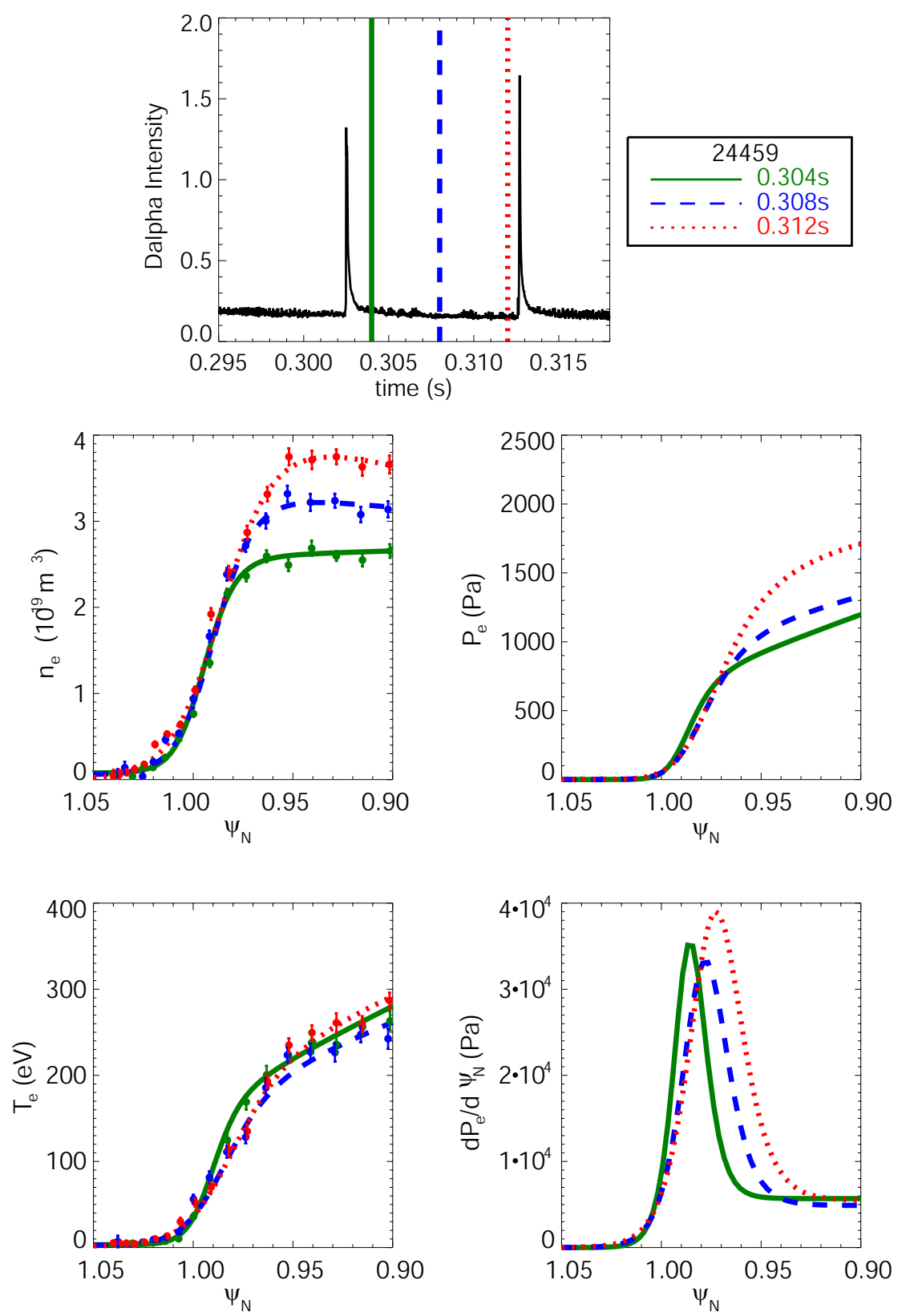

Figure 1: Profiles obtained during a single ELM cycle, showing the observed electron temperature and electron density data points in the pedestal, fitted profiles and the inferred electron pressure and electron pressure gradient profiles. 


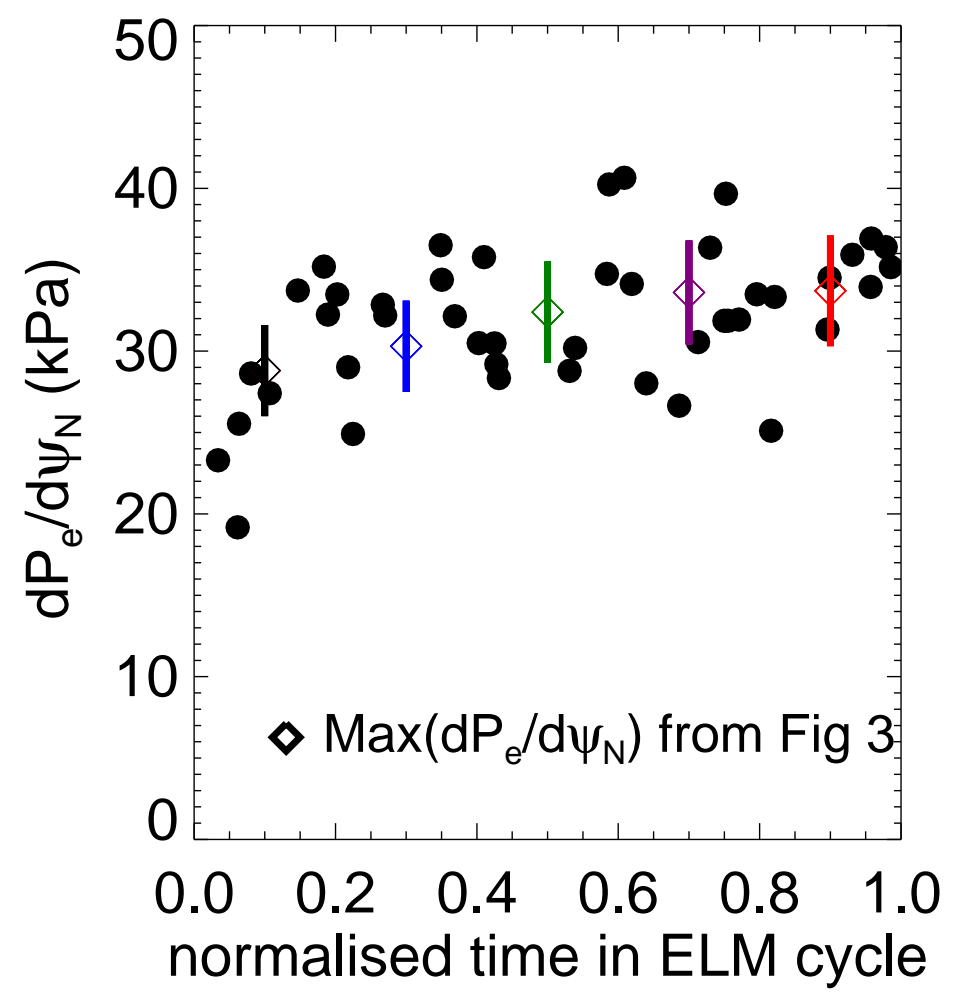

Figure 2: Evolution of the peak electron pressure gradient during the ELM cycle. The circles show the results from $\approx 50$ profile measurements. The diamonds show the peak pressure gradient from the fitted profiles shown in figure 3 . 

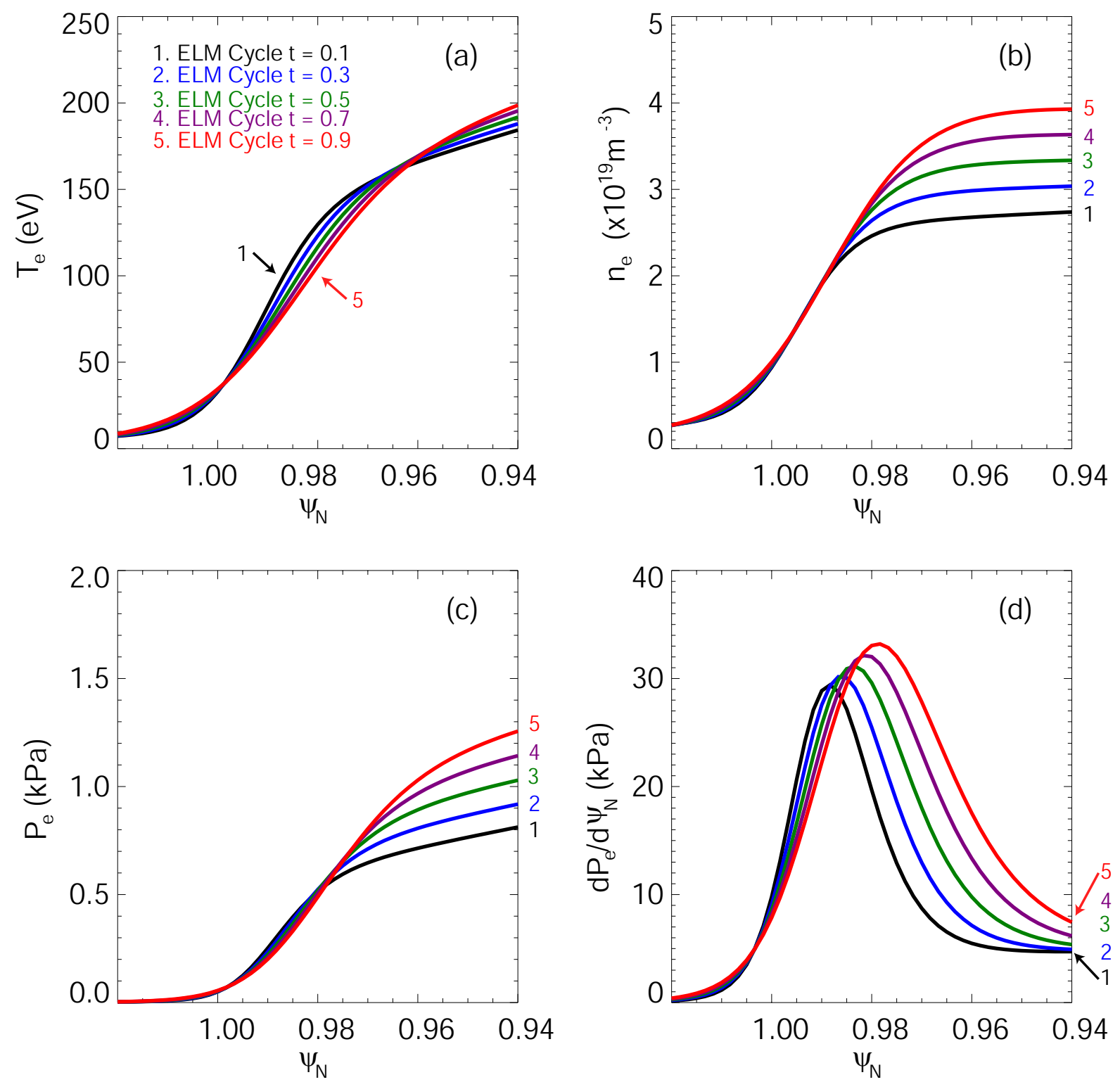

Figure 3: Evolution over the ELM cycle of: (a) electron temperature, (b) electron density, (c) electron pressure and (d) electron pressure gradient. Profiles were obtained from the mtanh fit parameters evaluated at the following normalised times during the ELM cycle: $t=(0.1,0.3,0.5,0.7,0.9)$. 


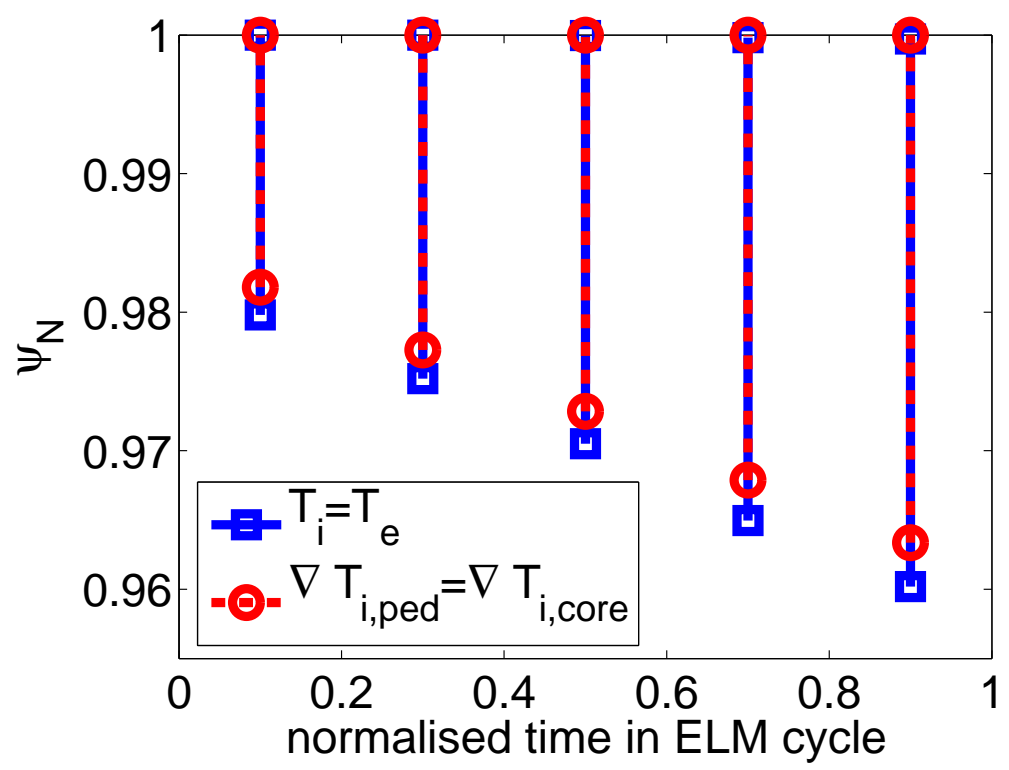

Figure 4: The unstable region for $n=\infty$ ballooning modes as a function of the normalised relative time in the ELM cycle for assumptions $T_{i}=T_{e}$ (blue squares, solid line) and $\nabla T_{i, \text { ped }}=\nabla T_{e, \text { core }}($ red circles, dashed line $)$. 


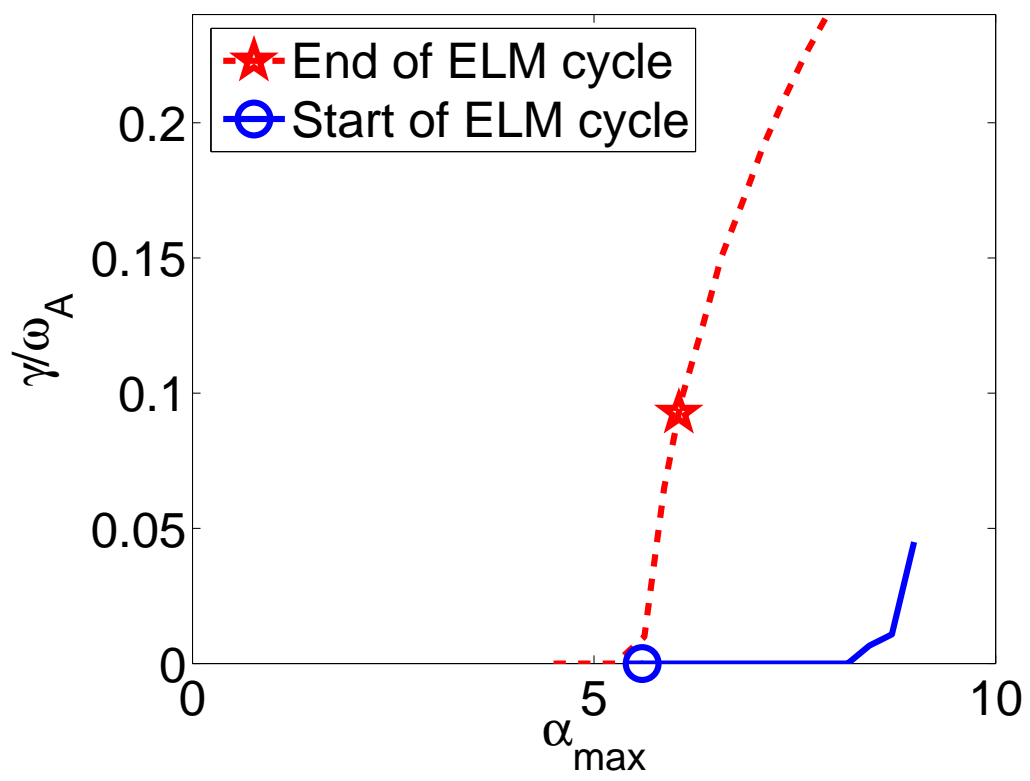

Figure 5: The growth rate of the most unstable mode, $n=25$, as a function of the maximum value of the normalised pressure gradient, $\alpha_{\max }$ for equilibria from the first (solid blue) and last (dashed red) intervals of the ELM cycle. The circle and the star show the experimental equilibrium values of $\alpha_{\max }$. Growth rates are normalised to the Alfven frequency in the centre of the plasma $\left(\omega_{A}\right)$. 


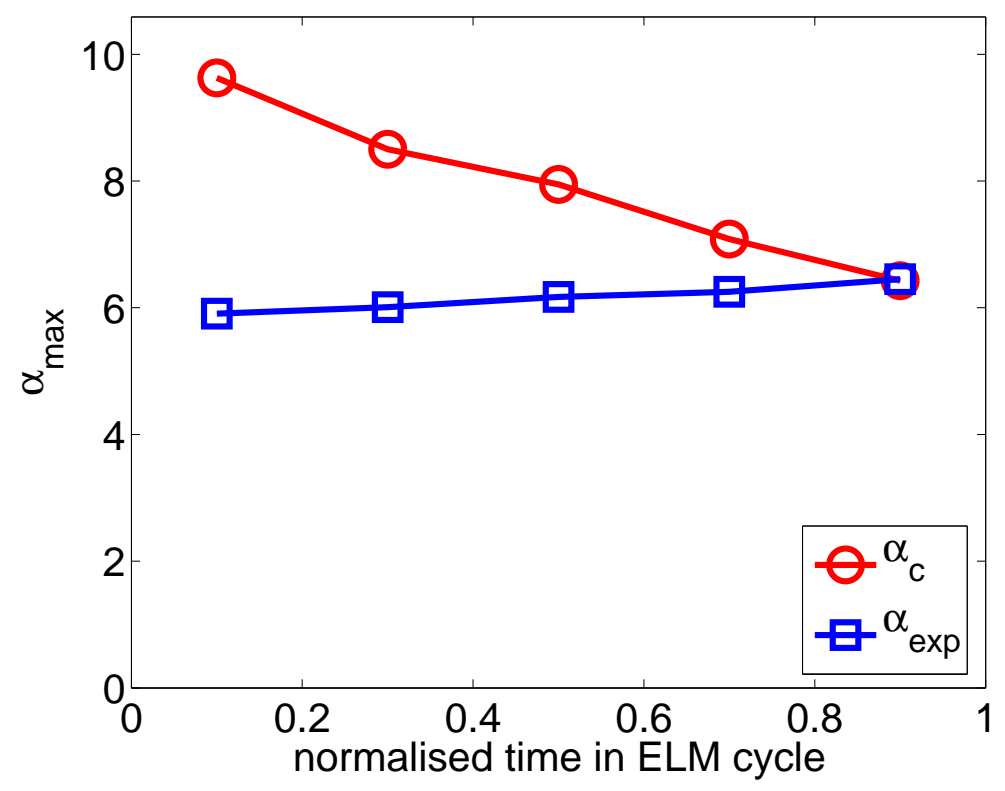

Figure 6: The maximum normalised pressure gradient in the experimental pedestal, $\alpha_{\exp }$, and the finite $n$ stability limit, $\alpha_{\mathrm{c}}$, as functions of the normalised time through the ELM cycle. 


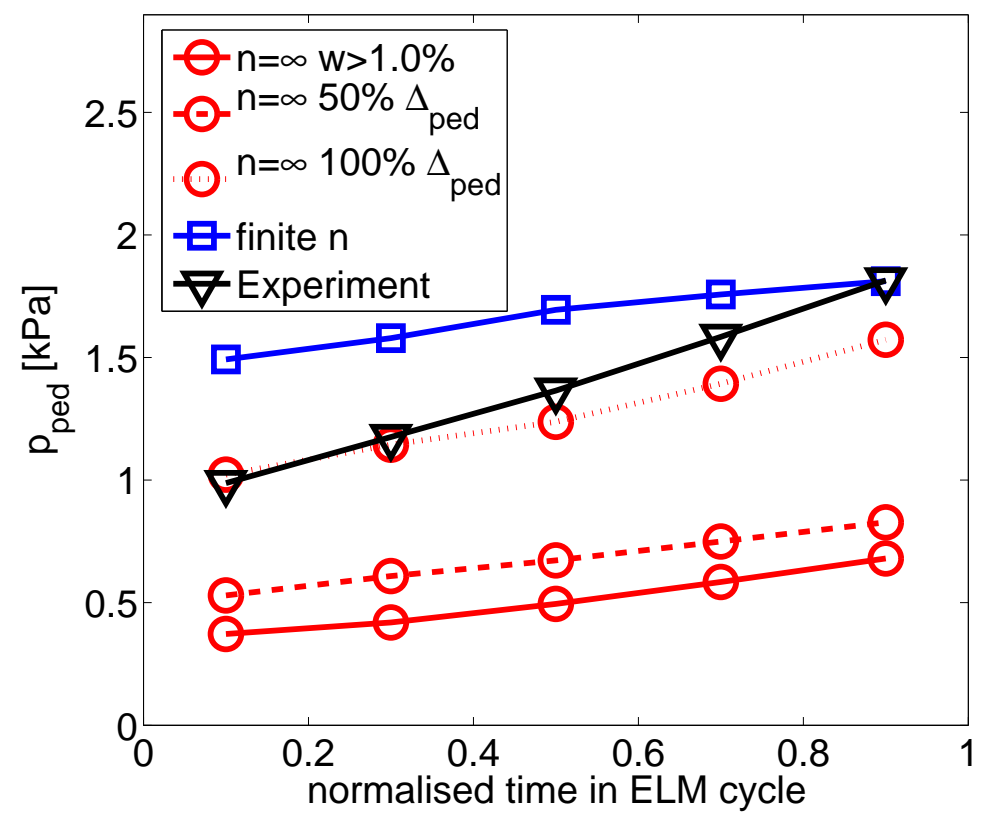

Figure 7: The pedestal top pressure of the experiment (black) and for profiles limited by the finite $n$ stability limit (blue), and the infinite-n ideal ballooning mode limit using three criteria: $1 \%$ of the total poloidal flux is unstable (solid red), $50 \%$ of the pedestal width is unstable (dashed red) and $100 \%$ of the pedestal width is unstable (dotted red). 


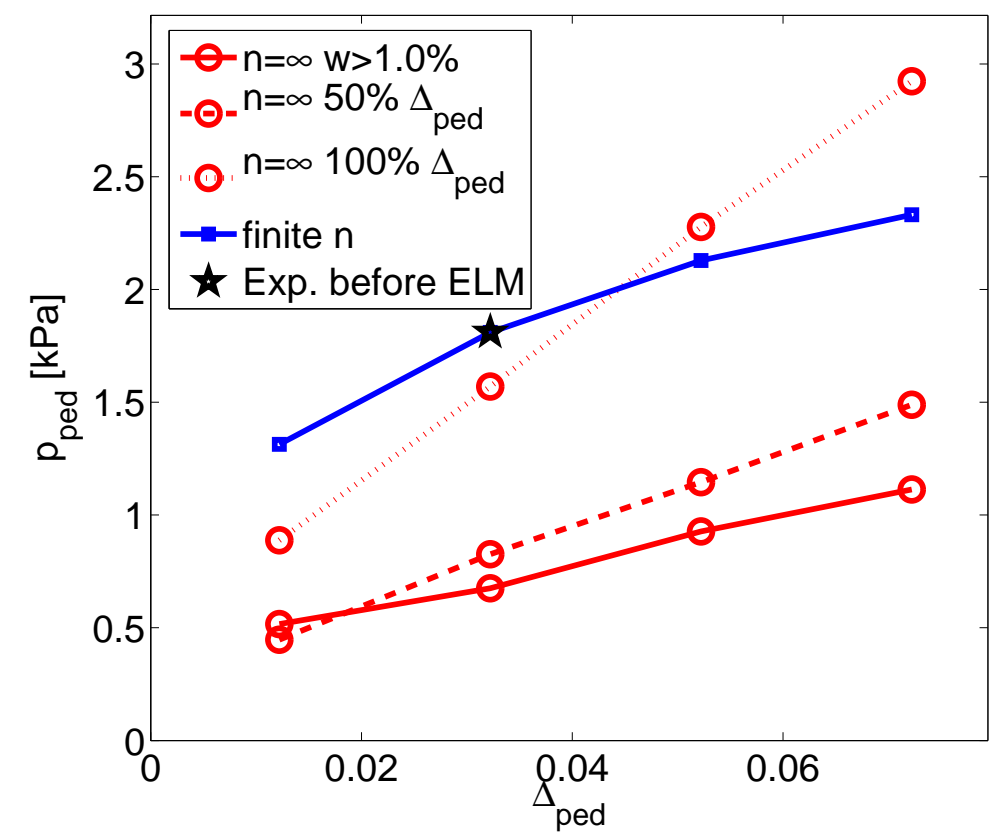

Figure 8: The pedestal top pressure as a function of the pedestal width for profiles limited by the finite- $n$ stability limit (blue), and the infinite-n ideal ballooning mode limit using three criteria: $1 \%$ of the poloidal flux is unstable (solid red), $50 \%$ of the pedestal width is unstable (dashed red), and $100 \%$ of the pedestal width is unstable (dotted red). 

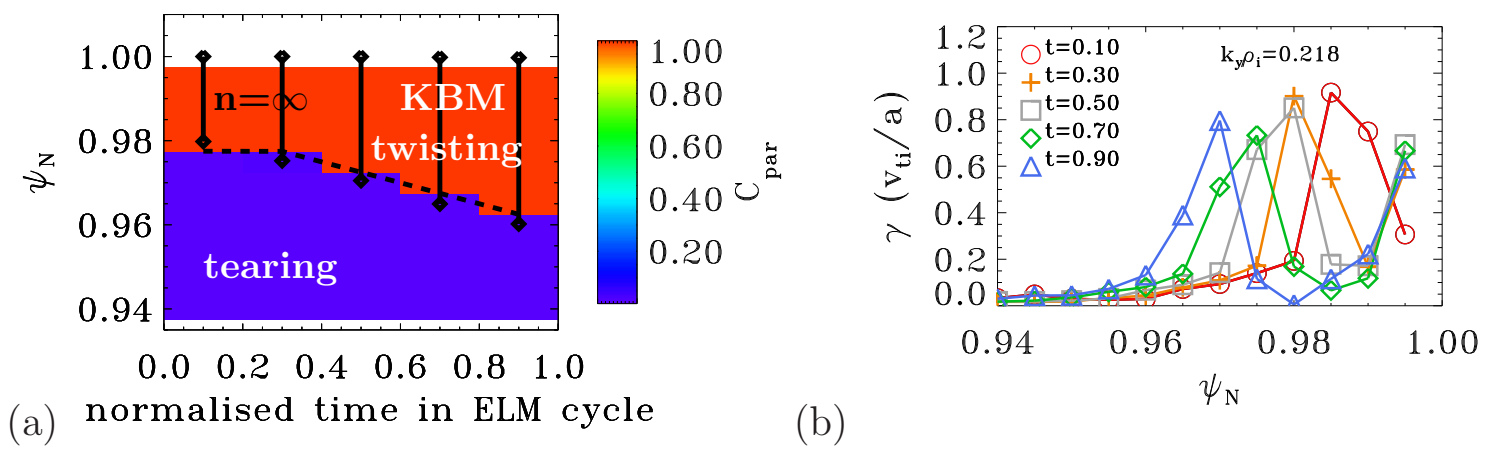

(b)

Figure 9: (a) Parity factor, $C_{\text {par }}$, of the dominant mode in range $0.07<k_{y} \rho_{i}<5.5$, and (b) growth rate of the fastest growing mode at $k_{y} \rho_{i}=0.218$, are both shown as functions of $\Psi_{N}$ and normalised time through the ELM cycle. In (a) the infinite-n ideal ballooning unstable region is illustrated by black lines and diamonds, and the boundary between the regions where twisting and tearing parity modes dominate is indicated by the dashed line. In (b) $\gamma$ is normalised to $v_{t i} / a$, where $v_{t i}$ is the local ion thermal velocity and $a$ is the minor radius. 


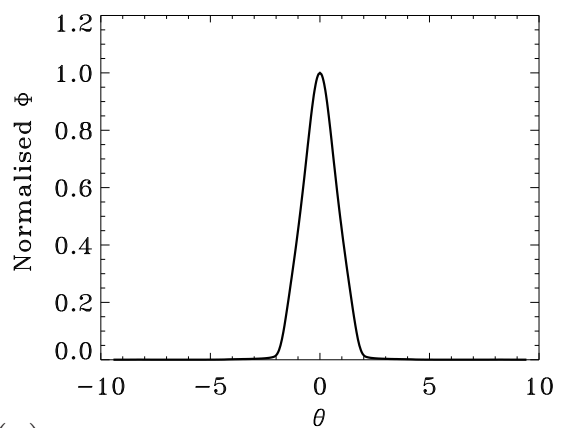

(a)

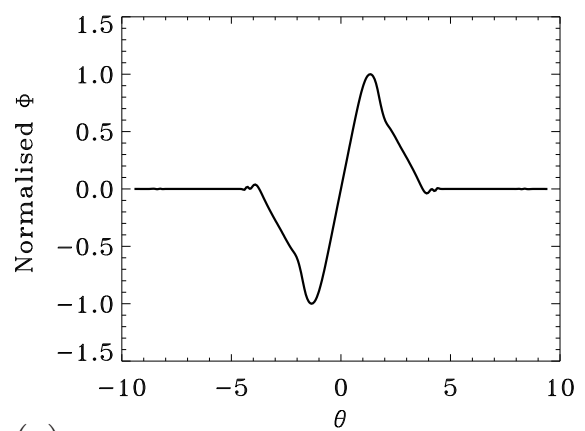

(c)

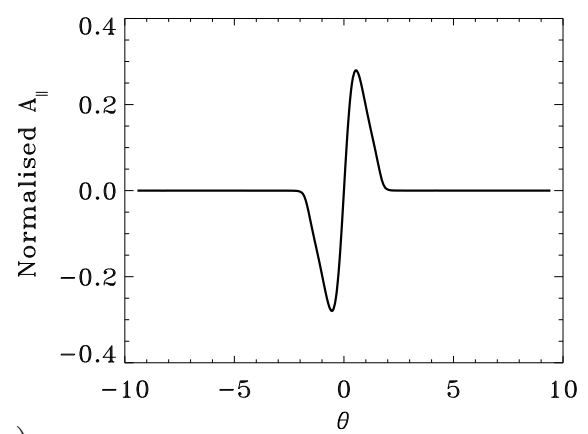

(b)

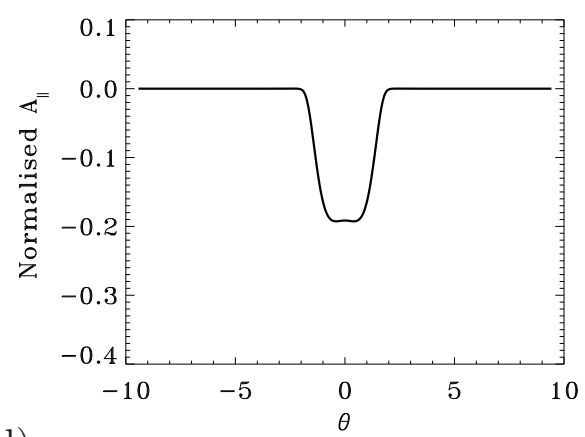

(d)

Figure 10: Normalised real part of the $\Phi$ and $A_{\|}$eigenfunctions for the dominant mode at the normalised ELM cycle time of 0.5 on surfaces $\Psi_{N}=0.98$ and $\Psi_{N}=0.95$. $\Phi$ and $A_{\|}$eigenfunctions are plotted as functions of ballooning angle, $\theta$, and are normalised to $\Phi\left(\theta_{\max }\right)$ and $\Phi\left(\theta_{\max }\right) / v_{t i}$, respectively, where $|\Phi|$ peaks at $\theta=\theta_{\max }$. On the outer surface, $\Psi_{N}=0.98$, the dominant mode has $k_{y} \rho_{i}=0.218$, and (a) $\Phi$ and (b) $A_{\|}$exhibit twisting parity. On the inner surface, $\Psi_{N}=0.95$, the dominant mode has $k_{y} \rho_{i}=3.28$, and (c) $\Phi$ and (d) $A_{\|}$have tearing parity. 